\title{
PERITONITIS EN PACIENTES QUE RECIBEN DIÁLISIS PERITONEAL EN UN HOSPITAL DE LIMA, PERÚ
}

\author{
Vanessa Pineda-Borja (1D) 1,a, Carolina Andrade-Santiváñez (iD) 1,a, Gustavo Arce-Gomez (iD) \\ Cristian León Rabanal (iD) ${ }^{1, b}$ \\ ${ }^{1}$ Universidad Peruana Cayetano Heredia, Lima, Perú. \\ ${ }^{\mathrm{a}}$ Médica/o cirujana/o; ${ }^{\mathrm{b}}$ Médico nefrólogo.
}

\section{RESUMEN}

Con el objetivo de determinar la tasa y factores relacionados con peritonitis en pacientes de una unidad de diálisis peritoneal (DP) atendidos en un hospital de Lima, Perú, se realizó un estudio retrospectivo de cohorte única durante el periodo de 2014 a 2016. Se incluyó a todos los pacientes con diagnóstico de enfermedad renal crónica del programa de DP del Hospital Cayetano Heredia. Se estimó la tasa de incidencia de peritonitis y se realizó un análisis bivariado para evaluar los factores relacionados. Se incluyó un total de 73 pacientes. La tasa de incidencia de peritonitis fue de 0,60 episodios por paciente al año; el $46,7 \%$ de los cultivos fueron positivos siendo el germen aislado más común el $S$. aureus. Los factores relacionados con peritonitis fueron menor valor de albúmina y hematocrito. La tasa de incidencia de peritonitis y de cultivos negativos se encontraron por encima del valor sugerido internacionalmente.

Palabras clave: Peritonitis; Diálisis Peritoneal; Factores de Riesgo (Fuente: DeCs BIREME).

\section{PERITONITIS IN PERITONEAL DIALYSIS PATIENTS FROM A HOSPITAL IN LIMA, PERU}

\begin{abstract}
In order to determine the rate and factors related to peritonitis in peritoneal dialysis (PD) patients treated at a hospital in Lima, Peru; a retrospective single-cohort study was conducted during the period 20142016. All patients diagnosed with chronic renal disease from the PD program at the Cayetano Heredia Hospital were included. The incidence rate of peritonitis was estimated and a bivariate analysis was performed to assess related factors. A total of 73 patients were included. The incidence rate of peritonitis was 0.60 episodes per patient per year; $46.7 \%$ of the cultures were positive and the most common isolated germ was $S$. aureus. Lower albumin and hematocrit values were found to be related to peritonitis. In this study, the incidence rate of peritonitis and negative cultures were found to be higher than the internationally estimated rate.
\end{abstract}

Keywords: Peritonitis, Peritoneal Dialysis, Risk Factors (Source: MeSH NLM).

\section{INTRODUCCIÓN}

En el Perú la frecuencia de pacientes en terapia de reemplazo renal (TRR) es de 415 pacientes por millón de población. De la población en TRR, el 88\% de pacientes se encuentran en hemodiálisis crónica (HDC), y el 12\%, en diálisis peritoneal (DP) ${ }^{(1)}$. La DP es un tipo de TRR tan efectiva como la HDC en relación con la sobrevida y, además, demuestra mejor calidad de vida relacionada con la salud durante el periodo inicial en comparación con la HDC, incluso ajustado para variables clínicas y socioeconómicas ${ }^{(2)}$.

La peritonitis es una complicación en la DP que constituye la primera causa de falla de esta técnica, retiro de catéter peritoneal y pase a HDC ${ }^{(3)}$. Se han reportado posibles factores relacionados con el desarrollo de peritonitis en pacientes en DP, los cuales incluyen obesidad, hipoalbuminemia, depresión, mayor distancia geográfica entre la residencia del paciente y la unidad de diálisis, técnica de diálisis (falta de entrenamiento, limitada bioseguridad), intervenciones invasivas (colonoscopias, histeroscopias), infecciones de orificio de salida del catéter peritoneal, colonización nasofaríngea de $S$. aureus, entre otros ${ }^{(3)}$.

En términos de supervivencia de la técnica y el estado de salud, la DP automatizada no ha demostrado ser superior a la DP continua ambulatoria ${ }^{(4,5)}$; sin embargo, en cuanto al riesgo relativo de peritonitis asociada a DP, los resultados son contradictorios ${ }^{(3,4,6)}$. Para un programa 
de DP es necesario determinar la frecuencia de complicaciones infecciosas, las frecuencias locales de peritonitis, el perfil microbiológico y el patrón de resistencia para guiar la práctica clínica en cuanto a tratamiento y pronóstico ${ }^{(7)}$. En la actualidad existe escasa información pública sobre los procedimientos o las complicaciones en los programas de DP de nuestro medio.

Así, el objetivo principal de este estudio es determinar la tasa de incidencia de peritonitis y los factores de riesgo relacionados con este en pacientes adultos de un programa de DP de Lima, Perú entre 2014 y 2016.

\section{EL ESTUDIO}

Se realizó un estudio longitudinal retrospectivo de cohorte única. Se incluyó a todos los pacientes mayores de 18 años, con diagnóstico de enfermedad renal crónica (ERC) y que se encontraran en el programa de DP del Hospital Cayetano Heredia en Lima, Perú, entre enero de 2014 y diciembre de 2016. Se excluyeron los pacientes que iniciaron tratamiento en otro centro de DP o que no tuvieran registrada información necesaria, es decir, valores de laboratorio en los últimos tres meses. Se excluyeron tres pacientes por este motivo.

Se consideró como episodio de peritonitis a aquellos pacientes que presentaran al menos dos de los siguientes criterios recomendados por la International Society Peritoneal Dialysis (ISPD) ${ }^{(3)}$ : a) características clínicas relacionadas con peritonitis (dolor abdominal o efluente peritoneal turbio); b) leucocitos en efluente peritoneal $>100 / \mu \mathrm{L}$, con $>50 \%$ polimorfonucleares $(\mathrm{PMN})$; c) cultivo positivo de efluente peritoneal. Se clasificó el episodio de peritonitis de acuerdo a la terminología establecida por la ISPD como "recurrencia», «recaída», «repetición»y «aislado» ${ }^{(3)}$.

Se recolectó información demográfica, clínica y de laboratorio registrada por el programa de DP e historias clínicas. Respecto de las variables del paciente, se registraron datos de edad, sexo, procedencia, grado de instrucción, empleo, ingresos, etiología de la ERC, comorbilidad, índice de masa corporal (IMC), tipo de DP, persona que realiza la diálisis, tiempo en DP, número de hospitalizaciones por cualquier motivo, meses desde la última hospitalización, valor de albúmina sérica, valor de hematocrito y destino del paciente después del episodio de peritonitis.

Para los episodios de peritonitis se registraron el tiempo en DP, la celularidad del líquido peritoneal (leucocitos y células polimorfonucleares), el resultado del cultivo, el tipo de peritonitis, los microorganismos aislados, la sensibilidad antibiótica, el tratamiento antibiótico, la infección del orificio de salida del catéter peritoneal, el valor de albúmina sérica y el hematocrito más cercano al momento del diagnóstico, IMC y destino. Se utilizaron los valores registrados al momento del diagnóstico de peritonitis.

Se utilizaron medidas de resumen y dispersión según la distribución de los datos obtenidos, y se compararon entre los grupos de pacientes con peritonitis y sin peritonitis. Se determinó la tasa de incidencia de peritonitis por paciente al

\section{MENSAJES CLAVE}

Motivación para realizar el estudio: La población que recibe diálisis está en aumento; sin embargo, existe escasa información sobre las complicaciones asociadas a diálisis peritoneal en nuestro medio.

Principales hallazgos: La tasa de incidencia de peritonitis en pacientes en diálisis peritoneal fue 0,60 episodios por pacienteaño. La proporción de cultivos negativos fue 53,3\%. Ambas se encuentran por encima de lo sugerido internacionalmente. Los factores relacionados a la peritonitis fueron un menor valor de albúmina y de hematocrito.

Implicancias: Se necesitan protocolos que estandaricen la recolección y análisis de fluido peritoneal; asimismo, se requieren estudios con mayor población y tiempo de seguimiento.

año, la tasa de incidencia por paciente al mes, la proporción de cultivos positivos y de cultivos negativos. El análisis de factores relacionados a la peritonitis asociada a la diálisis peritoneal fue evaluado por episodio de peritonitis, en lugar de participante, debido a ello, algunos episodios se presentan en el mismo paciente, y por tanto se repiten las medidas de variable específicas del paciente. Se compararon los grupos de pacientes con peritonitis y sin peritonitis mediante un análisis bivariado: chi cuadrado para las variables cualitativas, $t$ de Student para las variables cuantitativas con distribución paramétrica y la suma de rangos de Wilcoxon para las variables cuantitativas de distribución no paramétrica.

El proyecto del estudio fue previamente aprobado por el Comité de Ética de la Universidad Peruana Cayetano Heredia. Se analizó la información sin identificadores personales para preservar la confidencialidad del paciente.

\section{HALLAZGOS}

Se incluyó un total de 73 pacientes. La mediana de edad de los pacientes fue 39,0 años (RIQ 22-59), el 58,9\% de los pacientes fueron mujeres y el 67,6\% vivía en Lima. El 38,2\% de los pacientes tenía secundaria completa, la etiología de ERC más frecuente fue glomerulonefritis crónica con el 27,8\%. La comorbilidad más frecuente fue hipertensión arterial con el 56,0\%, la modalidad de diálisis más frecuente fue manual $(72,2 \%)$, realizada en la mayoría de casos $(58,6 \%)$ por el mismo paciente.

Las medias y desviación estándar (DE) de albúmina sérica en los pacientes que desarrollaron peritonitis $(3,19 \mathrm{mg} / \mathrm{dL}$; DE 0,74$)$ y en los pacientes que no desarrollaron peritonitis $(3,58 \mathrm{mg} / \mathrm{dL}$; DE 0,44$)$ tuvieron una diferencia significativa ( $p=0,041$ ); de igual manera las medias de hematocrito en los pacientes que desarrollaron $(30,39 \%$; DE 7,02$)$ y aquellos que no desarrollaron peritonitis $(34,39 \%$; DE 6,35) tuvieron una diferencia significativa $(\mathrm{p}=0,032)$ (Tabla 1$)$. 
Tabla 1. Características de los pacientes del programa de diálisis del Hospital Cayetano Heredia (2014-2016)

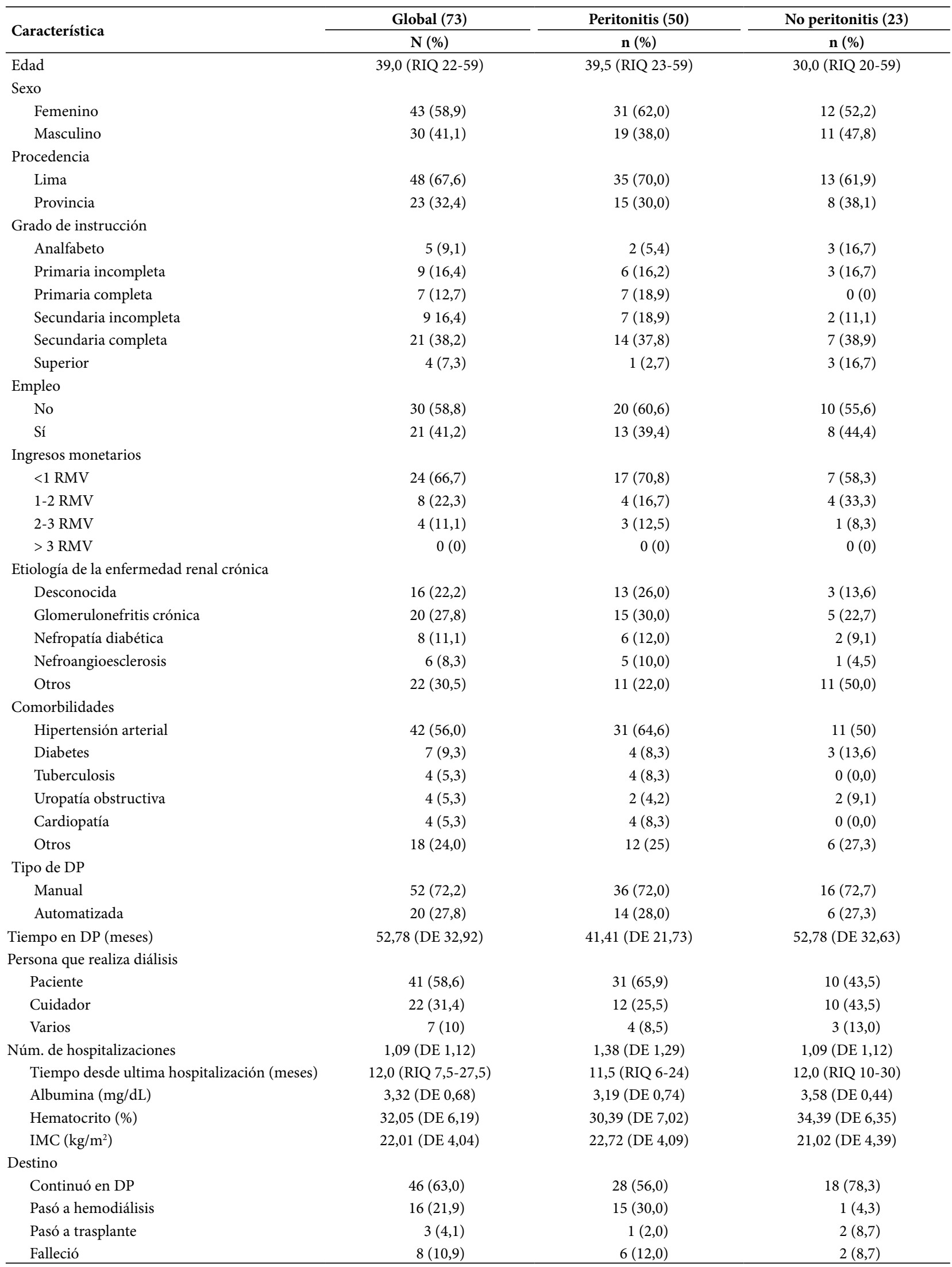

RIQ: rango inter cuartil, DE: desviación estándar, RMV: remuneración mínima vital, DP: diálisis peritoneal. 
El tiempo en diálisis promedio fue de 52,8 meses (DE $32,9)$. Los pacientes tuvieron en promedio 1,1 hospitalizaciones (DE 1,1) (Tabla 1). Al término del periodo de estudio, el 63,0\% de los pacientes continuaron en diálisis peritoneal.

Se registró un total de 114 episodios de peritonitis. Cincuenta pacientes $(68,5 \%)$ desarrollaron al menos un episodio de peritonitis durante el periodo de estudio. La tasa de incidencia de peritonitis fue 0,60 episodios por paciente al año y 0,05 episodios por paciente al mes ( 1 episodio cada 20 meses-paciente), otras características de los episodios de peritonitis según el paciente, la infección y el tratamiento se presentan en la Tabla 2.

Se obtuvo datos de los cultivos en 107 de los episodios de peritonitis, 50 de los cuales (46,7\%) eran cultivos positivos. Se reportó Staphylococcus aureus en 14 (28\%) episodios, Staphylococcus coagulasa negativo en 12 (24\%) cultivos y Candida sp. en 8 (16\%) cultivos. No se reportaron cultivos polimicrobianos, la resistencia antibiótica en los gérmenes aislados se presentan en el material complementario (Anexo 1).

\section{DISCUSIÓN}

La tasa de incidencia de peritonitis encontrada (0,60 episodios por paciente-año) supera la recomendación de la ISPD que es de menos de 0,50 episodios por paciente-año ${ }^{(3)}$. Este hallazgo es similar al reportado en el mismo programa de DP en población pediátrica $\left(0,61\right.$ episodios paciente-año) ${ }^{(8)}$, y es comparable a las tasas de incidencia de peritonitis de otros países de Latinoamérica, que reportan entre 0,35 a 0,80 episodios por paciente-año ${ }^{(9-12)}$. Tasas considerablemente bajas se han reportado en los Estados Unidos con 0,37 episodios por paciente-año ${ }^{(13)}$, y en China con 0,17 episodios por paciente-año ${ }^{(14)}$. Esta heterogeneidad puede ser explicada por la diferencia entre países del porcentaje de pacientes en TRR tratados con DP, particularmente en México, Estados Unidos, y China donde las políticas gubernamentales favorecen la DP.

La etiología de ERC más frecuente encontrada fue glomerulonefritis crónica $(27,8 \%)$, hallazgo similar al reportado por estudios realizados en un centro único de $\mathrm{DP}{ }^{(8,10,15)}$, en contraste a múltiples estudios que demuestran que la nefropatía diabética es la principal causa de ERC ${ }^{(1,2)}$. Esto podría ser explicado debido al pequeño tamaño de muestra, $\mathrm{y}$ a que se analizaron únicamente pacientes en DP.

Se encontró una alta tasa de episodios con cultivo negativo $(53,3 \%)$, la cual podría estar relacionada con el método de procesamiento no estandarizado de las muestras del líquido peritoneal o con el inicio de terapia antibiótica previo a la toma de muestra. Esto demuestra la necesidad de establecer protocolos de recolección y análisis de muestras de fluido peritoneal en todo el país. Un mismo laboratorio evaluó las muestras de líquido peritoneal, pero no necesariamente con
Tabla 2. Características según los episodios de peritonitis en pacientes del programa de diálisis del Hospital Cayetano Heredia (2014-2016)

\begin{tabular}{|c|c|}
\hline Características & n (\%) \\
\hline Total de episodios & 114 \\
\hline 2014 & $33(28,9)$ \\
\hline 2015 & $37(32,5)$ \\
\hline 2016 & $44(38,6)$ \\
\hline \multicolumn{2}{|l|}{ Sexo } \\
\hline Femenino & $75(65,8)$ \\
\hline Masculino & $39(34,2)$ \\
\hline \multicolumn{2}{|l|}{ Tipo de DP } \\
\hline Manual & $74(65,5)$ \\
\hline Automatizada & $39(34,5)$ \\
\hline Meses en DP & 25,50 (RIQ 17-38) \\
\hline \multicolumn{2}{|l|}{ Características de líquido } \\
\hline Leucocitos inicial (células/uL) & $1100 \mathrm{Leu} / \mathrm{uL}$ (RIQ 420-2800) \\
\hline Polimorfonucleares (\%) & 70 (RIQ 60-80) \\
\hline \multicolumn{2}{|l|}{ Resultado de cultivo } \\
\hline Negativo & $57(53,3)$ \\
\hline Positivo & $50(46,7)$ \\
\hline \multicolumn{2}{|l|}{ Infección del orificio } \\
\hline Sí & $6(5,5)$ \\
\hline No & $104(94,5)$ \\
\hline \multicolumn{2}{|l|}{ Tipo de peritonitis } \\
\hline Aislada & $107(97,3)$ \\
\hline Recaída & $1(0,9)$ \\
\hline Recurrencia & $0(0,0)$ \\
\hline Repetición & $3(2,7)$ \\
\hline \multicolumn{2}{|l|}{ Destino } \\
\hline Continuó en DP & $92(80,7)$ \\
\hline Pasó a hemodiálisis & $15(13,2)$ \\
\hline Pasó a trasplante renal & $1(0,9)$ \\
\hline Falleció & $6(5,3)$ \\
\hline \multicolumn{2}{|l|}{ Tratamiento } \\
\hline Ceftazidima & $94(82,5)$ \\
\hline Vancomicina & $96(84,2)$ \\
\hline Amikacina & $5(4,4)$ \\
\hline Ciprofloxacino & $2(1,7)$ \\
\hline Imipenem & $3(2,6)$ \\
\hline Meropenem & $3(2,6)$ \\
\hline Fluconazol & $2(1,7)$ \\
\hline
\end{tabular}

la misma máquina, lo cual puede introducir sesgo de medición; sin embargo, es probable que sea un sesgo no diferencial en naturaleza.

Staphylococcus aureus fue el germen aislado con mayor frecuencia, lo cual concuerda con un estudio previo realizado en el Perú en $2007{ }^{(16)}$. Este microorganismo ha sido 
asociado a episodios de mayor severidad, mayor riesgo de hospitalización, retiro de catéter, e incluso muerte ${ }^{(17)}$.

Además, se encontró relación entre un menor valor de hematocrito y el desarrollo de peritonitis. En los escasos estudios que han evaluado esta variable no se ha encontrado relación estadísticamente significativa con el desarrollo de peritonitis ${ }^{(18,19)}$.

A pesar de ser un factor de riesgo reconocido ${ }^{(3)}$, no se consideró la variable de colonización nasofaríngea previa o concomitante debido a que no todos los pacientes contaban con dicho registro.

No se halló relación entre la modalidad de diálisis y el desarrollo de la peritonitis. Algunos estudios sugieren que el riesgo relativo sería menor en la modalidad automatizada ${ }^{(4,6)}$; sin embargo, la mayoría de los estudios reportan resultados contradictorios.

No se encontró relación entre bajos ingresos o bajo nivel de educación y el desarrollo de peritonitis, lo cual difiere con lo encontrado en Australia ${ }^{(20)}$, Estados Unidos ${ }^{(13)}$, y Taiwán ${ }^{(15)}$. Se debe considerar que en el presente estudio dos tercios de la población observada se encuentra en pobreza, lo cual podría haber influido sobre el resultado.

En el programa de DP evaluado se realizan sesiones de entrenamiento de no más de 2 horas de duración por sesión, por un espacio de 15 días, acumulando un total de 30 horas. Asimismo, se realizan sesiones de reentrenamiento cada 6 meses, o cuando el paciente haya presentado un episodio de peritonitis. Se realizan evaluaciones, además de visitas de seguimiento tras la colocación del catéter peritoneal, o por complicaciones reportadas (peritonitis). A pesar de contar con el registro de los resultados de la evaluación escrita y de las visitas domiciliarias, estos instrumentos no han sido validados para evaluar la eficiencia del entrenamiento, y por ello no se incluyeron en el análisis.

Dada la naturaleza del estudio, es posible que existan variables intervinientes no consideradas en nuestro análisis o no capturadas por el programa. Otra limitación podría haber sido introducida por la exclusión de pacientes con registro incompleto del análisis, generando así un sesgo de selección. El pequeño número de pacientes que no desarrollaron peritonitis limitó la posibilidad de un diseño analítico. Se sugiere realizar estudios posteriores del tipo longitudinales analíticos, con mayor población y tiempo de seguimiento para determinar asociación y temporalidad entre los factores mencionados y el desarrollo de peritonitis en la población de estudio.

En conclusión, la tasa de incidencia de peritonitis y los cultivos positivos encontrados en el programa de diálisis peritoneal evaluado en un hospital de Lima, Perú, se encontraron por encima de los valores sugeridos internacionalmente. Los factores relacionados con el desarrollo de peritonitis fueron el menor valor de hematocrito y menor valor de albúmina sérica.

Contribución de los autores: VPB, CAS, GAG y CLR concibieron, diseñaron y redactaron el manuscrito; recolectaron, analizaron e interpretaron los resultados; hicieron la revisión crítica y aprobaron la versión final.

Fuentes de financiamiento: Autofinanciado.

Conflictos de interés: Los autores niegan tener algún conflicto de interés que pueda afectar la objetividad del presente trabajo.

Material suplementario: Disponible en la versión electrónica de la RPMESP.

\section{REFERENCIAS BIBLIOGRÁFICAS}

1. Ministerio de Salud, Análisis de la situación de la enfermedad renal crónica en el Perú, 2015. [Internet]. Lima: Dirección General de Epidemiología, MINSA; 2015 [citado el 14 de agosto del 2019]. Disponible en: https://www.dge.gob.pe/portal/index.php?option=com_content\&view $=$ article\&id $=598 \&$ Itemid $=353$.

2. Jung H, Jeon Y, Park Y, Kim YS, Kang SW, Yang CW, et al. Better Quality of Life of Peritoneal Dialysis compared to Hemodialysis over a Two-year Period after Dialysis Initiation. Sci Rep. 2019;9(1):10266. doi: 10.1038/s41598-019-46744-1.

3. Li PK, Szeto CC, Piraino B, de Arteaga J, Fan S, Figuereido AE, et al. International Society Peritoneal Dialysis peritonitis recommendations: 2016 update on prevention and treatment. Perit Dial Int. 2016;36(5):481-508. doi: 10.3747/pdi.2016.00078.

4. Balasubramanian G, McKitty K, Fan SL. Comparing automated peritoneal dialysis with continuous ambulatory peritoneal dialysis: survival and quality of life differences?. Nephrol Dial Transplant. 2011;26(5):1702-8. doi: 10.1093/ndt/gfq607.

5. Michels WM, Verduijn M, Boeschoten EW, Dekker FW, Krediet RT, NECOSAD Study Group. Similar Survival on Automated Peritoneal Dialysis and Continuous Ambulatory Peritoneal Dialysis in a Large Prospective Cohort. Clin J Am Soc Nephrol. 2009;4(5):943-9. doi: 10.2215/CJN.04440908.

6. Li X, Xu H, Chen N, Ni Z, Chen M, Chen L, et al. The Effect of Automated Versus Continuous Ambulatory Peritoneal Dialysis on
Mortality Risk In China. Perit Dial Int. 2018;38(Suppl 2):S25-S35. doi: 10.3747/pdi.2017.00235.

7. Ghali JR, Bannister KM, Brown FG, Rosman JB, Wiggins KJ, Johnson DW, et al. Microbiology and outcomes of peritonitis in Australian peritoneal dialysis patients. Perit Dial Int. 2011;31(6):651-62. doi: 10.3747/pdi.2010.00131.

8. Bernuy J, Cieza J. Tipos de membrana peritoneal y su sobrevida en función al test de equilibrio peritoneal en pacientes en DPCA. Rev Med Hered. 2010;21(1):11-17.

9. Nieto-Ríos JF, Díaz-Betancur JS, Arbeláez-Gómez M, García-García Á, Rodelo-Ceballos J, Reino-Buelvas A, et al. Peritoneal dialysis-related peritonitis: twenty-seven years of experience in a Colombian medical center. Nefrologia. 2014;34(1):88-95. doi: 10.3265/Nefrologia.pre2013.Nov.12002.

10. Figuereido AE, Poli-de-Figuereido CE, Meneghetti F, Pachecho GA, Costa C, Silva LB. Peritonitis in patients on peritoneal dialysis: analysis of a single Brazilian center based on the International Society for Peritoneal Dialysis. J Bras Nefrol 2013;35(3):214-219. doi: 10.5935/01012800.20130034.

11. Gadola L, Gomez T, Saez L, Perez D, Orihuela L, et al. Diez años del Registro Uruguayo de Peritonitis en Diálisis peritoneal. Rev Med Urug. 2016; 32(3):166-177.

12. Ramos A, Madonia C, Rascon-Pacheco RA. Improved patient/ technique survival and peritonitis rates in patients treated with automated peritoneal dialysis when compared to continuous ambulatory 
peritoneal dialysis in a Mexican PD center. Kidney Int Suppl. 2008; (108):S76-80. doi: 10.1038/sj.ki.5002606.

13. Kumar VA, Sidell MA, Yang WT, Jones JP. Predictors of peritonitis, hospital days, and technique survival for peritoneal dialysis patients in a managed care setting. Perit Dial Int. 2014;34(2):171-8. doi: 10.3747/pdi.2012.00165.

14. Tian Y, Xie X, Xiang S, Yang X, Zhang X, Shou Z, et al. Risk factors and outcomes of high peritonitis rate in continuous ambulatory peritoneal dialysis patients: A retrospective study. Medicine (Baltimore). 2016;95(49):e5569. doi: 10.1097/MD.0000000000005569.

15. Chern YB, Ho PS, Kuo LC, Chen JB. Lower education level is a major risk factor for peritonitis incidence in chronic peritoneal dialysis patients: a retrospective cohort study with 12-year follow-up. Perit Dial Int. 2013;33(5):55-2. doi: 10.3747/pdi.2012.00065.

16. Pecoits-Filho R, Abensur H, Cueto-Manzano AM, Dominguez J, Divino Filho JC, Fernandez-Cean J, et al. Overview of peritoneal dialysis in Latin America. Perit Dial Int. 2007;27(3):316-21. doi: $10.1177 / 089686080702700321$.
17. Barretti P, Moraes T, Camargo C, Caramori J, Mondelli A, Montelli $\mathrm{AC}$, et al. Peritoneal dialysis-related peritonitis due to Staphylococcus aureus: a single-center experience over 15 years. PloS One. 2012;7(2):e31780. doi: 10.1371/journal.pone.0031780.

18. Fan X, Huang R, Wang J, Ye H, Guo Q, Yi C et al. Risk factors for the first episode of peritonitis in Southern Chinese continuous ambulatory peritoneal dialysis patients. PloS One. $2014 ; 9(9)$ :e107485. doi: 10.1371/journal.pone.0107485.

19. Hsieh Y, Chang C, Wen Y, Chiu P, Yang Y. Predictors of peritonitis and the impact of peritonitis on clinical outcomes of continuous ambulatory peritoneal dialysis patients in Taiwan-10 years' experience in a single center. Perit Dial Int. 2014;34(1):85-94. doi: 10.3747/ pdi.2012.00075.

20. Tang W, Grace B, McDonald S, Hawley C, Badve S, Boudville NC, et al. Socio-Economic Status and Peritonitis in Australian Non-Indigenous Peritoneal Dialysis Patients. Perit Dial Int. 2015 ;35(4):450-9. doi: 10.3747/pdi.2013.00004. 